\title{
The changing morphology of the radio outflow of HESS J0632+057 along its orbit
}

\section{B. Marcote*}

Departament d'Astronomia i Meteorologia, Institut de Ciències del Cosmos, Universitat de Barcelona

E-mail: bmarcote@am.ub.es

\section{J. Moldón}

ASTRON Netherlands Institute for Radio Astronomy, Dwingeloo, The Netherlands

E-mail: moldon@astron.nl

\section{Ribó}

Departament d'Astronomia i Meteorologia, Institut de Ciències del Cosmos, Universitat de Barcelona

E-mail: mribo@am.ub.es

\section{J. M. Paredes}

Departament d'Astronomia i Meteorologia, Institut de Ciències del Cosmos, Universitat de Barcelona

E-mail: jmparedes@ub.edu

\section{Z. Paragi}

Joint Institute for VLBI in Europe, Dwingeloo, The Netherlands

E-mail: paragi@jive.nl

The gamma-ray binary HESS J0632+057 exhibits an orbitally modulated X-ray light-curve with a main and a secondary X-ray outburst. Previous EVN observations in 2011 just after the main $\mathrm{X}$-ray outburst reveal an extended radio emission and a decay on the total radio flux density. We observed the source around the secondary X-ray outburst at orbital phase $\phi=0.76$ with the EVN and simultaneously with WSRT to determine the changes of the radio emission at different scales. A radio outflow from this secondary outburst was expected. Two non-detections from the WSRT and EVN data point out a strong decrease of at least one order of magnitude in the radio emission with respect to the main X-ray outburst.

12th European VLBI Network Symposium and Users Meeting,

7-10 October 2014

Cagliari, Italy

\footnotetext{
*Speaker.
} 


\section{Introduction}

Gamma-ray binaries are binary star systems composed by a compact object and a young massive star showing the maximum of the Spectral Energy Distribution (SED) in the gamma-ray region (see [1] for a review). Only a handful of sources exhibiting these properties have been discovered up to now: PSR B1259-63, LS 5039, LS I +61 303, HESS J0632+057 and 1FGL J1018.6-5856. The first one is the only system hosting a confirmed pulsar. These systems are excellent laboratories to study high-energy processes, such as particle acceleration, emission and radiation reprocessing, and dynamics of the underlying magnetized flows.

The gamma-ray emission of these systems is probably produced by inverse Compton upscattering of stellar UV photons by relativistic electrons, which are thought to be accelerated by the shock between the relativistic wind of a young non-accreting pulsar and the wind of the stellar companion [1]. The radio spectrum is produced by synchrotron emission from the lower energy electrons. Extended emission at milliarcsecond (mas) scales has been reported for nearly all gamma-ray binaries at GHz frequencies [2]. The gamma-ray binaries PSR B1259-63, LS 5039, and LS I +61 303 show a radio morphology with a central core and one-sided extended radio emission on scales of a few $\mathrm{AU}$, although bipolar extended emission has also been detected at some orbital phases [3, 4]. This emission exhibits morphological changes along the orbital period, and the peak of the emission presents displacements larger than the orbit size [2].

\section{HESS J0632+057}

HESS J0632+057 was discovered by the High Energy Stereoscopic System (H.E.S.S.) Collaboration as a point-like, variable, $\mathrm{TeV}$ source [5, 6]. A variable $\mathrm{X}$-ray counterpart [6, 7] and also a variable radio counterpart [8] were found later. The massive B0 pe star MWC 148 was confirmed as the optical counterpart of the TeV source [7]. Recent X-ray observations with Swift/XRT have provided an improved orbital period of $315_{-4}^{+6}$ days [9]. The X-ray emission shows a main outburst in the orbital phase range $0.3-0.4$ followed by an X-ray dip at orbital phases $0.43-0.46$, and a secondary outburst between orbital phases 0.6 and 0.9 (phase $\phi=0$ is arbitrarily defined at MJD 54857 according to [10], while periastron takes place at orbital phase 0.97 following [11]). The maximum of the two X-ray outbursts changes slightly in phase and flux from cycle to cycle, while the X-ray dip remains stable. We note that HESS J0632+057 has not still been detected at GeV energies, making the system unique among all the known gamma-ray binaries [12]. The orbital variability of the $\mathrm{TeV}$ emission is correlated with that of the X-ray emission. This fact suggests that X-rays are produced by synchrotron emitting electrons that upscatter $\mathrm{UV}$ radiation from the companion to produce the observed $\mathrm{TeV}$ emission, supporting leptonic models [9].

The radio emission of HESS J0632+057 has been explored with connected interferometers (the Very Large Array, VLA, and the Giant Metrewave Radio Telescope, GMRT) at 1.3 and $5.0 \mathrm{GHz}$, mainly during the main X-ray outburst [8]. Two observations with the European VLBI Network (EVN) have been conducted in 2011 revealing extended emission at mas scales [13].

The SED of the source is very similar to the one of LS I +61303 , but one order of magnitude fainter [7]. We note that the distance to HESS J0632+057 is smaller than to LS I +61 303, implying that the source is intrinsically fainter. 

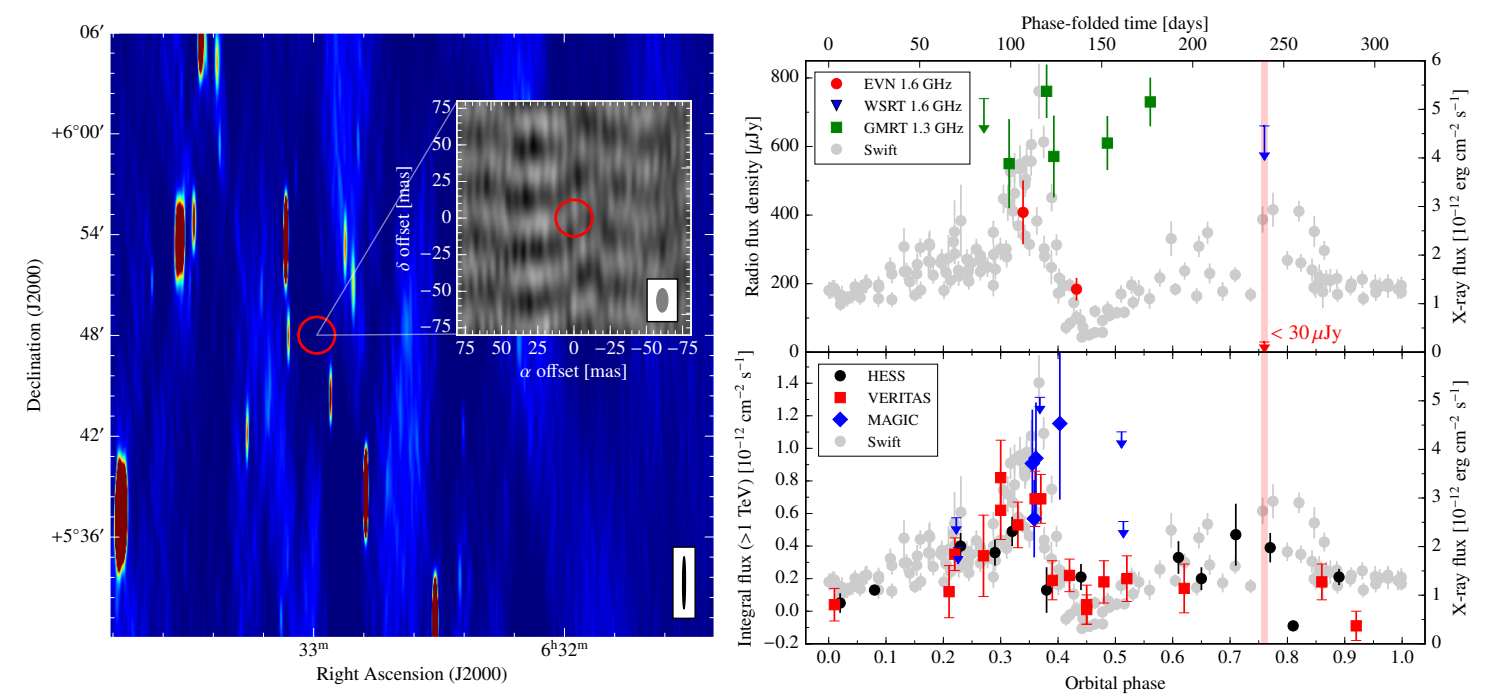

Figure 1: (left) image of the field of HESS J0632+057 from the WSRT data and the EVN one in the zoom. Radio emission from the source is not detected in any of both. (right) light-curve of HESS J0632+057 as a function of the orbital phase. The radio emission is shown on top and $\mathrm{TeV}$ emission on bottom. The gray circles represent the X-ray emission, the arrows represent the 3- $\sigma$ upper-limits, and the vertical pale red line denotes the orbital phase at which the presented observations took place.

\section{Observations and results}

We conducted a 10-hr EVN observation with the full array (Ef, Jb, Kn, Wb, Mc, On, Tr, Sh, $\mathrm{Ur}, \mathrm{Hh}, \mathrm{Sv}, \mathrm{Zd}, \mathrm{Bd}$ and Ro) at $1.6 \mathrm{GHz}$ on $20 \mathrm{Feb} .2014$ (at $\phi=0.76$, during the secondary X-ray outburst). The data were correlated at JIVE. WSRT (Wb) data were also recorded separately to obtain an image sensitive to larger scales. Fig. 1-left shows the WSRT and EVN images around the field of HESS J0632+057. The source is not detected, with 3- $\sigma$ upper-limits of $\sim 0.7$ mJy beam ${ }^{-1}$ (WSRT) and $30 \mu \mathrm{Jy}^{\text {beam }}{ }^{-1}$ (EVN). Fig. 1-right shows the light-curve of HESS J0632+057 as a function of the orbital phase from the existing $\mathrm{X}$-ray, $\mathrm{TeV}$ and $1-2 \mathrm{GHz}$ radio data. The obtained upper-limits are shown at the orbital phase denoted by a vertical pale red line.

\section{Discussion}

The EVN non-detection reveals a strong decay in the radio emission of HESS J0632+057 of at least one order of magnitude respect to the one observed during the main X-ray outburst. However, from the X-ray behavior we would not expect such a strong decay. The X-ray-yo-radio flux radio around the main $\mathrm{X}$-ray outburst is $\sim 10^{-6}$. Considering the non-simultaneous $\mathrm{X}$-ray data from Swift and assuming this $\mathrm{X}$-ray emission during the EVN observation, this ratio is $\lesssim 10^{-7}$ during the secondary X-ray outburst. This reduction in the X-ray-to-radio flux ratio from one epoch to another could be explained by different behaviors. On one hand, the X-ray and radio emission can arise from a different electron population, naturally explaining the lack of correlation. On the other hand, the X-ray and radio data that we have compared are not simultaneous. Hence, we do not know the X-ray emission at the epoch when the radio observation was conducted. We note that the secondary X-ray outburst presents a large variability from cycle to cycle according to the Swift data [9], but in any case much lower than the observed decay in the radio flux density. 


\section{Conclusions}

The 2014 EVN upper-limit during the secondary X-ray outburst implies a strong decrease (of at least one order of magnitude) in the radio emission of HESS J0632+057 compared to the two EVN observations conducted in 2011 during the main X-ray outburst. Although variability from cycle to cycle has been reported in X-rays, the radio light-curve remains poorly sampled. This strong decrease could be periodic with the orbital phase or an effect produced only at some epochs. Simultaneous X-ray and radio observations must be conducted to understand the behavior of HESS J0632+057 at all orbital phases and to determine the possible connection between the $\mathrm{X}$-ray and radio emission.

Acknowledgments: B.M., M.R. and J.M.P. acknowledge support by the Spanish Ministerio de Economía y Competitividad (MINECO) under grants AYA2013-47447-C3-1-P and FPA2013-48381-C6-6-P. B.M. acknowledges financial support from MINECO under grant BES-2011-049886. J.M.P. acknowledges financial support from ICREA Academia. The EVN is a joint facility of European, Chinese, South African and other radio astronomy institutes funded by their national research councils.

\section{References}

[1] G. Dubus, Gamma-ray binaries and related systems, Astronomy and Astrophysics Rev. 21 (2013) 64

[2] J. Moldón, Structure and nature of gamma-ray binaries by means of VLBI observations, PhD Thesis, Universitat de Barcelona (2012)

[3] V. Dhawan, A. Mioduszewski, and M. Rupen, LS I +61 303 is a Be-Pulsar binary, not a Microquasar, VI Microquasar Workshop: Microquasars and Beyond (2006)

[4] J. Moldón, M. Ribó, and J. M. Paredes, Periodic morphological changes in the radio structure of the gamma-ray binary LS 5039, Astronomy and Astrophysics 548 (2012) 103

[5] F. A. Aharonian, A. G. Akhperjanian, A. R. Bazer-Bachi, et al., Discovery of a point-like very-high-energy $\gamma$-ray source in Monoceros, Astronomy and Astrophysics 469 (2007) 1-4

[6] V. A. Acciari, E. Aliu, T. Arlen, et al., Evidence for Long-Term Gamma-Ray and X-Ray Variability from the Unidentified TeV Source HESS J0632+057, Astrophysical Journal Letters 698 (2009) 94-97

[7] J. A. Hinton, J. L. Skilton, S. Funk, et al., HESS J0632+057: A New Gamma-Ray Binary?, Astrophysical Journal Letters 690 (2009) 101-104

[8] J. L. Skilton, M. Pandey-Pommier, J. A. Hinton, et al., The radio counterpart of the likely TeV binary HESS J0632+057, Monthly Notices of the RAS 399 (2009) 317-322

[9] E. Aliu, S. Archambault, T. Aune, et al., Long-term TeV and X-Ray Observations of the Gamma-Ray Binary HESS J0632+057, Astrophysical Journal Letters 780 (2014) 168

[10] S. D. Bongiorno, A. D. Falcone, M. Stroh, et al., A New TeV Binary: The Discovery of an Orbital Period in HESS J0632+057, Astrophysical Journal Letters 737 (2011) 11

[11] J. Casares, M. Ribó, I. Ribas, et al., On the binary nature of the $\gamma$-ray sources AGL J2241+4454 (= MWC 656) and HESS J0632+O57 (= MWC 148), Monthly Notices of the RAS 421 (2012) 1103-1112

[12] G. A. Caliandro, A. B. Hill, D. F Torres, et al., The missing GeV $\gamma$-ray binary: searching for HESS J0632+057 with Fermi-LAT, Monthly Notices of the RAS 436 (2013) 740-749

[13] J. Moldón, M. Ribó, and J. M. Paredes, Revealing the extended radio emission from the gamma-ray binary HESS J0632+057, Astronomy and Astrophysics 533 (2011) L7 